\title{
SOME THEOREMS ON INTEGRAL FUNCTIONS WITH NEGATIVE ZEROS
}

\author{
BY \\ N. A. BOWEN AND A. J. MACINTYRE
}

1. In this paper we answer a question raised by Titchmarsh concerning integral functions whose zeros are real and negative, and whose asymptotic behaviour on the negative real axis is prescribed. In particular we prove the following result.

\section{THEOREM I. Let}

$$
f(z) \equiv \prod_{n=1}^{\infty}\left(1+\frac{z}{a_{n}}\right) \quad\left(0<a_{n}<a_{n+1}, n=1,2,3, \cdots\right)
$$

be an integral function such that, for each $\epsilon>0$,

$$
\log |f(-x)|<\pi x^{\rho}(\cot \pi \rho+\epsilon)
$$

for all sufficiently large $x(>0)$, and

$$
\log \left|f\left(-x_{n}\right)\right|>\pi x_{n}^{\rho}(\cot \pi \rho-\epsilon)
$$

for sufficiently large $x_{n}$ of a sequence such that

$$
x_{n} \rightarrow+\infty, \quad x_{n+1} / x_{n} \rightarrow 1 .
$$

Then, as $x$ tends to infinity along the positive real axis, the relation

$$
\log f(x) \sim \pi x^{\rho} \csc \pi \rho
$$

holds if either

(i) $0<\rho<1 / 2$ and

$$
\log f(x)=o\left(x^{1 / 2}\right) ; \text { or }
$$

(ii) $1 / 2<\rho<1$.

Part (i) of this theorem was proved by Titchmarsh [6, p. 195] $]^{(1)}$, who pointed out that nothing can be expected if $\rho=1 / 2$, and left part (ii) as a speculation. Actually Titchmarsh replaces (5) of the hypothesis by the condition $n(x)=o\left(x^{1 / 2}\right)$, which is trivially equivalent to (5), and the conclusion by $n(x) \sim x^{\rho}$, which follows from (4) by a theorem of Tauberian character [1, pp. $90-95 ; 2$, pp. 210-212; 6, pp. 186-188; 7, pp. 237-243; 8, pp. 121-127].

We find it desirable to take up a more general viewpoint similar to that of Paley and Wiener [5, p. 79, Theorem XXVI], considering Theorem I as

Received by the editors March 13, 1950.

(1) Numbers in brackets refer to the references at the end of the paper. 
a kind of limiting case of the following theorem.

Theorem II. Let $|\alpha|<\pi, 0<j<1$, and the function $f(z)$ defined by (1) satisfy

$$
\log \left|f\left(r_{n} e^{i \alpha}\right)\right|<\pi r_{n}^{p} \csc \pi \rho(\cos \rho \alpha+\epsilon)
$$

for sufficiently large $r_{n}$ of a sequence such that

$$
r_{n} \rightarrow+\infty, \quad r_{n+1} / r_{n} \rightarrow 1,
$$

and satisfy

$$
\log \left|f\left(t_{n} e^{i \alpha}\right)\right|>\pi t_{n}^{\rho} \csc \pi \rho(\cos \rho \alpha-\epsilon)
$$

for a similar sequence $t_{n}$. Then, as $x$ tends to infinity along the positive real axis, the relation

$$
\log f(x) \sim \pi x^{\rho} \csc \pi \rho
$$

holds if either

(8) (i)

$$
|\alpha|<\pi /(2 \rho)
$$

and

$$
\log f(x)=o\left(x^{\pi / 2|\alpha|}\right)
$$

or

(10) (ii)

$$
|\alpha|>\pi /(2 \rho) \text {. }
$$

Theorem II is not so to speak proved directly, but is obtained by a device, essentially due to Titchmarsh, from the following theorem.

THEOREM III. Let $|\alpha|<\pi, 0<\rho<1$, and the function $f(z)$ defined by (1) satisfy

$$
\log \left|f\left(r e^{i \alpha}\right)\right|<\pi r^{\rho} \csc \pi \rho(\cos \rho \alpha+\epsilon) \quad(r>r(\epsilon)) .
$$

Then

$$
\log f(x)<\pi x^{\rho}(\csc \pi \rho+\epsilon) \quad(x>x(\epsilon))
$$

follows from (8) and (9), and

$$
\log f(x)>\pi x^{\rho}(\csc \pi \rho-\epsilon) \quad(x>x(\epsilon))
$$

from (10).

If (11) is replaced by

$$
\log \left|f\left(r e^{i \alpha}\right)\right|>\pi r^{\rho} \csc \pi \rho(\cos \rho \alpha-\epsilon) \quad(r>r(\epsilon))
$$

then (13) follows from (8) and (9), and (12) from (10). 
Functions of the form (1) are of at most order 1. We are able, in Theorems IV, V, and VI, to give generalisations of Theorems III, II, and I respectively to functions of finite order, the first generalisation being in a sense complete, the others partial.

2. Our proof of Theorem III is based on the following lemma.

LEMMA 1. Let $\psi(z)$ be an analytic function of $z=r e^{i \theta}$, regular for $|\arg z|<\pi$, and on the negative real axis with the possible exception of logarithmic singularities.

Suppose also that $\psi(z)$ is real on the positive real axis and that

$$
\begin{aligned}
&|\psi(z)|=o\left(r^{p-1 / 2}\right) \text { as } \quad|z|=r \rightarrow 0, \\
& \int_{-\pi}^{\pi}\left|\psi\left(r e^{i \theta}\right)\right| d \theta=o\left(r^{p+1 / 2}\right) \quad \text { as } \quad r \rightarrow \infty
\end{aligned}
$$

where $p$ is an integer.

Then for $|\arg z|<\pi$ we have

$$
\psi(z)=\frac{(-1)^{p}}{\pi} \int_{0}^{\infty} \frac{z^{p+1 / 2} R\left\{\psi\left(t e^{i \pi}\right)\right\} d t}{t^{p+1 / 2}(t+z)}-\quad(z \neq 0) .
$$

Proof. The result is obtained by considering the contour integral

$$
\psi(z)=\frac{1}{2 \pi i} \int_{\Gamma} \frac{z^{p+1 / 2} \psi(\zeta) d \zeta}{\zeta^{p+1 / 2}(\zeta-z)},
$$

where $\Gamma$ is the contour formed by the radii $\arg \zeta= \pm \pi$ joined by the circumferences of the circles

$$
|\zeta|=\delta, \quad|\zeta|=R
$$

and $z$ lies within this contour. The integral along the first circle tends to zero as $\delta \rightarrow 0$, by (15), and the integral along the second tends to zero as $R \rightarrow \infty$, by (16). Hence

$$
\psi(z)=\frac{(-1)^{p}}{2 \pi i}\left[\int_{0}^{\infty} \frac{i z^{p+1 / 2}\left\{\psi\left(t e^{-i \pi}\right)+\psi\left(t e^{i \pi}\right)\right\} d t}{t^{p+1 / 2}(t+z)}\right]
$$

and since $\psi(z)$ is supposed to be real on the positive real axis, it takes conjugate values at conjugate points, and we have

$$
\psi(z)=\frac{(-1)^{p}}{\pi} \int_{0}^{\infty} \frac{z^{p+1 / 2} R\left\{\psi\left(t e^{i \pi}\right)\right\} d t}{t^{p+1 / 2}(t+z)}
$$

as required.

Proof of Theorem III. We may obviously assume that $\alpha>0$.

In Lemma 1 we put 


$$
\psi(z) \equiv \log f\left(z^{\alpha / \pi}\right),
$$

take $z=x$, and obtain the formula

$$
\log f\left(x^{\lambda}\right)=\frac{(-1)^{p}}{\pi} \int_{0}^{\infty} \frac{x^{p+1 / 2} \log \left|f\left(t^{\lambda} e^{i \alpha}\right)\right| d t}{t^{p+1 / 2}(t+x)}
$$

where

$$
\lambda=\alpha / \pi .
$$

Since we propose, by means of the inequality (11), to approximate to the above integral in terms of

$$
I \equiv \int_{0}^{\infty} \frac{x^{p+1 / 2} t^{\lambda \rho-p-1 / 2} d t}{(t+x)},
$$

we must choose the integer $p$ so as to satisfy the convergence requirements of (18), as well as (15) and (16). Now we know that

$$
0<\lambda \rho<1
$$

and since the integral (18) converges only when

$$
p-1 / 2<\lambda \rho<p+1 / 2 \text {, }
$$

the only possible values of the integer $p$ are 0 and 1 .

Near the origin

$$
|\psi(z)|=\left|\log f\left(z^{\lambda}\right)\right|=O\left(|z|^{\lambda}\right),
$$

and as $|z|$ tends to infinity

$$
|\psi(z)|=\left|\log f\left(z^{\lambda}\right)\right|=o\left(|z|^{\lambda}\right)\left({ }^{2}\right) .
$$

It is easily seen that when

$$
\lambda \rho<1 / 2
$$

we must take $p=0$ to satisfy (19). In this case (15) is a result of (20), and (16) of

$$
\left|\log f\left(z^{\lambda}\right)\right|=o\left(|z|^{1 / 2}\right),
$$

a condition that follows from

$$
\log f(x)=o\left(x^{\pi / 2 \alpha}\right)\left({ }^{2}\right) .
$$

(2) We here jústify (21) and, for convenience, also set down some results required later. From the convergence of $\prod_{n-1}^{\infty}\left(1+z / a_{n}\right) \quad\left(0<a_{n}<a_{n+1} ; n=1,2,3, \cdots\right)$ follows that of $\sum_{n=1}^{\infty} a_{n}^{-1}$ and $\int^{\infty} n(t) d t / t^{2}$. Also $n(x)=o(x), \log f(x)=\int_{0}^{\infty} x n(t) d t / t(t+x)=o(x)$. In $|\arg z| \leqq \pi-\delta$, $\delta>0$, wehave $|\log f(z)|=\left|\int_{0}^{\infty} z n(t) d t / t(t+z)\right| \leqq \csc (\delta / 2) \int_{0}^{\infty}|z| n(t) d t / t(t+|z|)=\csc (\delta / 2) \cdot \log f(|z|)$ $=o(|z|)$, since $|z+t|^{2} \geqq(t-|z| \cos \delta)^{2}+(|z| \sin \delta)^{2}=\left(t^{2}+|z|^{2}+2 t|z|\right)-2 t|z|(1+\cos \delta)$ $\geqq\{(t+|z|) \sin (\delta / 2)\}^{2}$. 
Again, when

$$
\lambda \rho>1 / 2
$$

we must take $p=1$. We now have $\lambda>1 / 2$, and so (15) still follows from (20), and (23) is satisfied by any function of the form $(1)\left({ }^{2}\right)$.

To evaluate $I$ let $t=x u$. Then

$$
\begin{aligned}
I & =x^{\lambda \rho} \int_{0}^{\infty} \frac{u^{\lambda \rho-p-1 / 2} d u}{(u+1)} \\
& =x^{\lambda \rho} \pi \csc \pi(\lambda \rho-p+1 / 2) \\
& =\pi x^{\lambda \rho} \sec \rho \alpha \\
& =-\pi x^{\lambda \rho} \sec \rho \alpha
\end{aligned}
$$

when $p=0$

when $p=1$.

When $p=0$ it follows from (11), (17), (18), and (26) that

$$
\log f\left(x^{\lambda}\right)<\pi x^{\lambda \rho}\left(\csc \pi \rho+\epsilon_{1}\right)
$$

which becomes (12) by replacing $x^{\lambda}, \epsilon_{1}$ by $x, \epsilon$ respectively. We note that (22) and (24) are the conditions numbered (8) and (9) in the enunciation of the theorem.

Similarly, when $p=1$, it is easily seen that (13) follows from (11), (17), (18), and (26), provided that (25), that is, (10) holds.

The second half of the theorem is proved in the same way $\left({ }^{3}\right)$.

Proof of Theorem II. In this case the asymptotic inequalities $\{(6)$ and (7) $\}$ are given only for discrete sets of points on arg $z=\alpha$, but, following Titchmarsh $[6$, p. 196], we can show that the corresponding inequalities are satisfied without exception on nearby radii arg $z=\alpha \pm \delta$, and the above argument, with $\alpha \pm \delta$ in place of $\alpha$, completes the proof of Theorem II.

Let $\alpha>\pi / 2, \beta=\pi-\alpha$. Then, with $\delta>0$ and $\alpha+\delta<\pi$,

$$
\log \left|f\left(R e^{i(\alpha+\delta)}\right)\right|<\log \left|f\left(r_{n} e^{-i \alpha}\right)\right|
$$

when

$$
\left|1-\frac{R e^{-2 i(\beta-\delta)}}{a_{n}}\right|^{2}<\left|1-\frac{r_{n} e^{i \beta}}{a_{n}}\right|^{2},
$$

that is, when

$$
R^{2}-r_{n}^{2}<2 a_{n}\left(R \cos \overline{\beta-\delta}-r_{n} \cos \beta\right),
$$

which is true for every $a_{n}$ if

(3) We have to thank the referee for the remark that of the four statements made in this theorem the two in which the inequality proceeds in the same way in hypothesis and conclusion follow from Phragmen-Lindelöf theorems. In the other two cases the angle is too large for the application of Phragmen-Lindelöf theorems, and our lemma seems essential. 


$$
r_{n} \frac{\cos \beta}{\cos \overline{\beta-\delta}} \leqq R \leqq r_{n} .
$$

For all such values of $R$ it follows from (27) and (6) that

$$
\begin{aligned}
\log \left|f\left(R e^{i(\alpha+\delta)}\right)\right| & <\pi r_{n}^{\rho} \csc \pi \rho(\cos \rho \alpha+\epsilon) \\
& <\pi R^{\rho} \csc \pi \rho\{\cos \rho(\alpha+\delta)+2 \epsilon\},
\end{aligned}
$$

where $\delta=\delta(\epsilon)$. Actually (29) holds for all large $R$, as required, since the hypothesis $r_{n} \rightarrow+\infty, r_{n+1} / r_{n} \rightarrow 1$ ensures that, for all $n$ and therefore $r_{n}$ sufficiently large, the intervals (28), corresponding to consecutive values of $n$, overlap.

A similar discussion, but with (7) in place of (6), leads to

$$
\log \left|f\left(R e^{i(\alpha-\delta)}\right)\right|>\pi R^{\rho} \csc \pi \rho\{\cos \rho(\alpha-\delta)-2 \epsilon\} .
$$

When $0<\alpha \leqq \pi / 2$, the corresponding inequalities are verified by the same technique, the details being easier, and for $\alpha=0,(29)$ and (30) with $\delta=0$ follow simply from (6), (7), and the fact that $f(x)$ increases with $x$. When $-\pi<\alpha<0$, we have only to observe that $|f(\bar{z})|=|f(z)|$.

Theorem II now follows at once from Theorem III, by using (29) and (30) in place of (11) and (14).

Proof of Theorem I. Here $\alpha=\pi$. (30) with $\alpha=\pi$ is obtained as above and we get one of the required inequalities at once by using Theorem III. The other inequality follows from (2) and Lemma 1 by the argument used in proving Theorem III. Here, however, we are applying the lemma for $\lambda \equiv \alpha / \pi$ $=1$, and we must verify that the condition (16), which becomes

$$
\int_{-\pi}^{\pi}\left|\log f\left(r e^{i \theta}\right)\right| d \theta=o\left(r^{p+1 / 2}\right)
$$

is satisfied in spite of the fact that $\log f(z)$ is infinite when $z=-a_{n}$, $n=1,2,3, \cdots$. We discuss the behaviour of the latter integral indirectly, by considering

$$
\begin{aligned}
& I_{1} \equiv \int_{-\pi}^{\pi}\left|\arg f\left(r e^{i \theta}\right)\right| d \theta, \\
& I_{2} \equiv \frac{1}{2 \pi} \int_{-\pi}^{\pi} \log ^{+}\left|1 / f\left(r e^{i \theta}\right)\right| d \theta,
\end{aligned}
$$

and

$$
I_{3} \equiv \frac{1}{2 \pi} \int_{-\pi}^{\pi} \log ^{+}\left|f\left(r e^{i \theta}\right)\right| d \theta
$$


where $\log ^{+} u$ means $\log u$ when $u>1$, and 0 otherwise.

Suppose now that

$$
\log f(x)=o\left(x^{\rho^{\prime}}\right)
$$

and hence $\left({ }^{4}\right)$

$$
n(x)=o\left(x^{\rho^{\prime}}\right)
$$

From

$$
\begin{aligned}
\arg f\left(r e^{i \theta}\right) & =\sum_{n=1}^{\infty} \arg \left(1+\frac{r e^{i \theta}}{a_{n}}\right) \\
& =\left\{\sum_{a_{n}<2 r}+\sum_{a_{n} \geqq 2 r} \arg \left(1+\frac{r e^{i \theta}}{a_{n}}\right)\right\},
\end{aligned}
$$

it follows, with $\arg f(z)=0$ on the positive real axis, that

$$
\begin{aligned}
\left|\arg f\left(r e^{i \theta}\right)\right| & \leqq\left\{\pi n(2 r)+\sum_{a_{n} \geqq 2 r} \sin ^{-1}\left(\frac{r}{a_{n}}\right)\right\} \\
& <\left\{\pi n(2 r)+2 r \sum_{a_{n} \geqq 2 r} a_{n}^{-1}\right\} \\
& \leqq\left\{\pi n(2 r)+2 r \int_{2 r}^{\infty} \frac{d n(t)}{t}\right\} \\
& =\left\{\pi n(2 r)+2 r \int_{2 r}^{\infty} \frac{n(t) d t}{t^{2}}\right\} \\
& =o\left(r^{\rho^{\prime}}\right)
\end{aligned}
$$

Thus

$$
I_{1}=o\left(\boldsymbol{r}^{\boldsymbol{\rho}^{\prime}}\right) \text {. }
$$

Now, $I_{2}, I_{3}$ are the means values denoted by $m(r, 1 / f), m(r, f)$ by Nevanlinna, who has shown $[3$, p. $158 ; 4$, p. 12$]$ that for integral functions

$$
m(r, f)=m(r, 1 / f)+N(r, 1 / f)+O(1)
$$

for all sufficiently large $r$, where $N$ is positive. It follows that

$$
\begin{aligned}
I_{2} \equiv m(r, 1 / f) & <m(r, f) \equiv I_{3} \\
& =o\left(r^{\rho^{\prime}}\right),
\end{aligned}
$$

From (32), (33), (34), (37), and (38) we have (31) as required, provided

(4) $o\left(x^{\rho^{\prime}}\right)=\log f(x) \equiv \int_{0}^{\infty} x n(t) d t / t(t+x)>\int_{x}^{\infty} x n(t) d t / t(t+x)>n(x) \log 2$.

(5) When $\rho^{\prime}=1,(35),(36)$, and the convergence of $\int^{\infty} n(t) d t / t^{2}$ are all consequences of the convergence of $\mathrm{II}_{n-1}^{\infty}\left(1+z / a_{n}\right)\left(0<a_{n}<a_{n+1} ; n=1,2, \cdots\right)$. Compare footnote 2 . 
that $\rho^{\prime} \leqq p+1 / 2$.

Thus we are justified in putting $\psi(z)=\log f\left(z^{\lambda}\right)$ in Lemma 1, even when $\lambda \equiv \alpha / \pi=1$. The main lines of the proof of Theorem I are now clear. The analogue of (17) is

$$
\log f(x)=\frac{(-1)^{p}}{\pi} \int_{0}^{\infty} \frac{x^{p+1 / 2} \log |f(-t)| d t}{t^{p+1 / 2}(t+x)}
$$

which, with (2), gives one of the required inequalities, by the argument used in proving Theorem III. The other inequality follows from Theorem III, since, by Titchmarsh's device $\left({ }^{6}\right)$, (3) ensures that $f(z)$ satisfies

$$
\log \left|f\left(r e^{i(\pi-\delta)}\right)\right|>\pi r^{\rho} \csc \pi \rho\{\cos \rho(\pi-\delta)-\epsilon\} \quad(\delta>0, r>r(\epsilon))
$$

which is the hypothesis (14) of Theorem III with $\alpha=\pi-\delta$.

3. Functions of higher order. It is natural to enquire whether corresponding theorems hold for similar integral functions of higher order, and only trivial modifications of the proof of Theorem III from Lemma 1 are needed to obtain the following theorem.

Theorem IV. Let $|\alpha|<\pi, q<\rho<q+1\left(^{7}\right)$ and the canonical product

$$
f(z) \equiv \prod_{n=1}^{\infty}\left(1+\frac{z}{a_{n}}\right) \exp \left(-\frac{z}{a_{n}}+\frac{1}{2} \cdot \frac{z^{2}}{a_{n}^{2}}-\cdots+\frac{(-1)^{q}}{q} \cdot \frac{z^{q}}{a_{n}^{q}}\right)
$$

of at most order $(q+1)$, convergent type, satisfy

$$
\log \left|f\left(r e^{i \alpha}\right)\right| \sim \pi r^{\rho} \csc \pi \rho \cos \rho \alpha .
$$

Then the relation

$$
\log f(x) \sim \pi x^{\rho} \csc \pi \rho
$$

holds if either

$$
\text { (i) } \quad 0<|\alpha|<\pi / 2 \rho \text { and } \log f(x)=o\left(x^{\pi / 2|\alpha|}\right) \text {; or }
$$

$$
\text { (ii) } \frac{(2 s-1) \pi}{2 \rho}<|\alpha|<\frac{(2 s+1) \pi}{2 \rho} \text { and } \log f(x)=o\left(x^{(2 s+1) \pi / 2|\alpha|}\right)
$$

with $s$ a positive integer.

We are not able to generalise Theorems II and I completely, but give partial generalisations in Theorems V and VI respectively. The loss of generality

(6) Used in proving Theorem II above.

( 7$)$ The restrictions on $\rho$ are necessitated by the following considerations. Firstly, the order of the canonical product (40) cannot exceed $(q+1)$, whence we must have $\rho \leqq q+1$ in (41). Secondly, by the definition of a canonical product, (40) implies divergence of $\sum_{n=1}^{\infty} a_{n}^{-q}$, so that $n(t) \geqq t^{q}$, giving $|\log f(x)| \equiv \int_{0}^{\infty} x^{q+1} n(t) d t / t^{q+1}(t+x) \geqq x^{q}$. Thus (42) is impossible if $\rho \leqq q$. Finally, integral values of $\rho$ must obviously be excluded from (41). 
is due to a further restriction on $\alpha$ in Theorem $\mathrm{V}$ and to a severe restriction on $\rho$ in Theorem VI. The latter theorem, however, together with Theorem I, part (ii), does show that Titchmarsh's original Theorem, where $0<\rho<1 / 2$, can be extended to cover the range $0<\rho<3 / 2, \rho \neq 1 / 2, \rho \neq 1$.

Theorem V. Let $|\alpha|<\pi, q<\rho<q+1$, and the function defined by (40) satisfy

$$
\log \left|f\left(r_{n} e^{i \alpha}\right)\right|<\pi r_{n}^{\rho} \csc \pi \rho\left(\cos \rho \alpha+(-1)^{q} \epsilon\right)
$$

for sufficienctly large $r_{n}$ of a sequence such that

$$
r_{n} \rightarrow+\infty, \quad r_{n+1} / r_{n} \rightarrow 1,
$$

and satisfy

$$
\log \left|f\left(t_{n} e^{i \alpha}\right)\right|>\pi t_{n}^{\rho} \csc \pi \rho\left(\cos \rho \alpha-(-1)^{q} \epsilon\right)
$$

for a similar sequence $t_{n}$. Then, as $x$ tends to infinity along the positive real axis, the relation

$$
\log f(x) \sim \pi x^{\rho} \csc \pi \rho
$$

follows from either of (43), (44) when $\cos q \alpha, \cos \overline{q+1} \alpha$ are not of opposite sign.

In order to prove this theorem we reduce it to Theorem IV by showing that the hypotheses enable us to verify that (45), (46) are valid for all large $r$ in place of $r_{n}$ and $t_{n}$. Titchmarsh's device for "filling up the gaps" is no longer available, but in Lemma 2 below we develop an alternative method available whenever

$$
n(t)<K t^{\rho}
$$

is known. (47) follows from (45) or (46) when $\cos q \alpha, \cos \overline{q+1} \alpha$ are not of opposite sign. It seems plausible that the last clause could be omitted from Theorem V, giving a complete generalisation of Theorem II.

Our proof of (47) proceeds as follows.

From (45) and the real part of the integral

$$
(-1)^{q} \int_{0}^{\infty} \frac{z^{q+1} n(t) d t}{t^{q+1}(t+z)}
$$

for $\log f(z)$, evaluated at $z=r_{n} e^{i \alpha}$, we have

$$
(-1)^{q} \int_{0}^{\infty} \frac{(u \cos \overline{q+1} \alpha+\cos q \alpha) n\left(r_{n} u\right) d u}{u^{q+1}\left(1+u^{2}+2 u \cos \alpha\right)}
$$

$$
<\pi r_{n}^{p} \csc \pi \rho\left(\cos \rho \alpha+(-1)^{q} \epsilon\right)
$$

after putting $t=r_{n} u$, and we remember that 


$$
q<\rho<q+1 .
$$

When $q$ is even and $\cos q \alpha, \cos \overline{q+1} \alpha$ are not negative, we find from (48) that

$$
n\left(r_{n}\right) \int_{1}^{\infty} \frac{(u \cos \overline{q+1} \alpha+\cos q \alpha) d u}{u^{q+1}\left(1+u^{2}+2 u \cos \alpha\right)}<\pi \dot{r}_{n} \csc \pi \rho(\cos \rho \alpha+\epsilon)
$$

and hence

$$
n\left(r_{n}\right)<K r_{n}^{p}
$$

Dealing similarly with other cases in which the integrand is of constant sign and choosing from (45) and (46) the appropriate inequality, we conclude that (49), with $t_{n}$ sometimes in place of $r_{n}$, holds whenever $\cos q \alpha$ and $\cos \overline{q+1} \alpha$ are not of opposite sign. In order to obtain (49) for all large $r$, in place of all large $r_{n}$, we recollect that

$$
r_{n} \rightarrow+\infty, \quad r_{n+1} / r_{n} \rightarrow 1,
$$$$
\text { as } n \rightarrow \infty
$$

and hence for

$$
r_{n} \leqq r \leqq r_{n+1},
$$

we have

$$
\begin{aligned}
\frac{n(r)}{r^{\rho}} \leqq \frac{n\left(r_{n+1}\right)}{r_{n}^{\rho}} & <\frac{n\left(r_{n+1}\right)(1+\epsilon)^{\rho}}{r_{n+1}^{\rho}} \\
& <K
\end{aligned}
$$

if $\epsilon$ is chosen sufficiently small. Thus the required inequality (47) holds for all large $t$.

The validity of (45) and (46) for all large $r$, in place of $r_{n}$ and $t_{n}$, now follows from the following lemma.

$$
\text { LeMma 2. Let }|\alpha|<\pi, q<\rho<q+1 \text {, }
$$

$$
n(t)<K t^{\rho}
$$

and

$$
\phi(z) \equiv z^{-\rho} \log f(z),
$$

where $f(z)$ is defined by (40). Then, on $\arg z=\alpha$,

$$
\frac{d\{\phi(z)\}}{d\{\log z\}}=\omega(z)
$$

is bounded.

We have 


$$
\left.\int_{z=r_{n} e^{i \alpha}}^{z=r e^{i \alpha}} d \phi(z)\right\}=\int_{z=r_{n} e^{i \alpha}}^{z=r e^{i \alpha}} \frac{\omega(z) d z}{z},
$$

that is,

$$
\begin{aligned}
\left|\phi\left(r e^{i \alpha}\right)-\phi\left(r_{n} e^{i \alpha}\right)\right| & \leqq\left(r-r_{n}\right) \max \left|\frac{\omega(z)}{z}\right| \\
& <K\left(\frac{r}{r_{n}}-1\right) \\
& \leqq K\left(\frac{r_{n+1}}{r_{n}}-1\right)
\end{aligned}
$$$$
\left(r_{n} \leqq r \leqq r_{n+1}\right)
$$

since $\omega(z)$ is bounded, and it follows from (52) that, if $r_{n}$ and $r_{n+1}$ are consecutive members of the sequence mentioned in Theorem $\mathrm{V}$, the inequalities

$$
\left|\phi\left(r e^{i \alpha}\right)-\phi\left(r_{n} e^{i \alpha}\right)\right|<\epsilon
$$

that is,

$$
\left|\frac{\log f\left(r e^{i \alpha}\right)}{\left(r e^{i \alpha}\right)^{\rho}}-\frac{\log f\left(r_{n} e^{i \alpha}\right)}{\left(r_{n} e^{i \alpha}\right)^{\rho}}\right|<\epsilon
$$

and a fortiori

$$
\left|r^{-\rho} \log \right| f\left(r e^{i \alpha}\right)\left|-r_{n}^{-\rho} \log \right| f\left(r_{n} e^{i \alpha}\right)||<\epsilon
$$

are true for all $n>n_{0}(\epsilon)$, and

$$
r_{n} \leqq r \leqq r_{n+1}
$$

We conclude that the inequality $(45)$ is valid for all large $r$. The same argument shows that it is permissible to replace $t_{n}$ in (46) by $r$, and this completes the reduction of Theorem V to Theorem IV. We must, however, give a proof of Lemma 2.

Proof of Lemma 2. From

$$
\phi(z) \equiv z^{-\rho} \log f(z)=(-1)^{q} z^{q+1-\rho} \int_{0}^{\infty} \frac{n(t) d t}{t^{q+1}(t+z)}
$$

we have

$$
\begin{aligned}
\frac{d\{\phi(z)\}}{d\{\log z\}}=(-1)^{q} z\left\{(q+1-\rho) z^{q-\rho} \int_{0}^{\infty} \frac{n(t) d t}{t^{q+1}(t+z)}\right. & \left.-z^{q+1-\rho} \int_{0}^{\infty} \frac{n(t) d t}{t^{q+1}(t+z)^{2}}\right\}
\end{aligned}
$$

When $z=r e^{i \alpha}$, with $|\alpha|<\pi$, 


$$
\begin{aligned}
|z+t|^{2} & =(t+r \cos \alpha)^{2}+(r \sin \alpha)^{2} \\
& =\left(t^{2}+r^{2}+2 t r\right)-2 t r(1-\cos \alpha) \\
& \geqq\{(t+r) \cos (\alpha / 2)\}^{2} .
\end{aligned}
$$

The boundedness of $d\{\phi(z)\} / d\{\log z\}$ follows from (47), (53), and (54). For example, the first integral in (53) is less in modulus than

$$
\begin{aligned}
& \sec (\alpha / 2) \int_{0}^{\infty} \frac{K t^{\rho-q-1} d t}{(t+r)}=K r^{\rho-q-1} \sec (\alpha / 2) \int_{0}^{\infty} \frac{u^{\rho-q-1} d u}{(u+1)} \\
& =K_{1} r^{p-q-1} \\
& (t=r u),
\end{aligned}
$$

since $q<\rho<q+1$, and the second is similarly less than $K_{2} r^{p-q-2}$.

We conclude this paper by stating and proving the following extension of Titchmarsh's theorem.

THEOREM VI. Let

$$
f(z) \equiv \prod_{n=1}^{\infty}\left(1+\frac{z}{a_{n}}\right) e^{-z / a_{n}} \quad\left(0<a_{n}<a_{n+1}, n=1,2,3, \cdots\right)
$$

be a canonical product such that, for each $\epsilon>0$,

$$
\log |f(-x)|<\pi x^{\rho}(\cot \pi \rho+\epsilon)
$$

for all sufficiently large $x(>0)$, and

$$
\log \left|f\left(-x_{n}\right)\right|>\pi x_{n}^{\rho}(\cot \pi \rho-\epsilon)
$$

for sufficiently large $x_{n}$ of a sequence such that

$$
x_{n} \rightarrow+\infty, \quad x_{n+1} / x_{n} \rightarrow 1 .
$$

Then, as $x$ tends to infinity along the positive real axis, the relation

$$
\log f(x) \sim \pi x^{\rho} \csc \pi \rho
$$

holds if $1<\rho<3 / 2$ and

$$
\log f(x)=o\left(x^{3 / 2}\right)
$$

as $x$ tends to infinity.

Proof of Theorem VI. We use the same argument as in the proof of Theorem I, but have to replace Titchmarsh's device $\left({ }^{8}\right)$ for filling up the gaps, by Lemma 2 . The deduction of

$$
-\log f(x)<\pi x^{\rho}(-\csc \pi \rho+\epsilon)
$$

follows at once from (55) and formula (39) with $p=1$. The opposite inequality

(8) Used in proving Theorem II above. 


$$
-\log f(x)>\pi x^{\rho}(-\csc \pi \rho-\epsilon),
$$

needed to complete the proof, is similarly deduced from

$$
\log \left|f\left(r e^{i(\pi-\delta)}\right)\right|>\pi r^{\rho} \csc \pi \rho\left\{\cos \rho(\pi-\delta)+\epsilon_{1}\right\}
$$

and formula (17) with $\lambda \equiv \alpha / \pi=(\pi-\delta) / \pi$.

We show that (58) is a consequence of the hypotheses as follows. Putting

$$
\log f(x)=-\int_{0}^{\infty} \frac{x^{2} n(t) d t}{t^{2}(t+x)}
$$

in (57), we have

$$
\int_{x}^{\infty} \frac{x^{2} n(t) d t}{t^{2}(t+x)}<\pi x^{\rho}(-\csc \pi \rho+\epsilon)
$$

whence

$$
n(x)<K x^{p} \text {. }
$$

Hence, by Lemma 2, (58) holds for all large $r$ if it does for a sequence $r_{n}$ such that $r_{n} \rightarrow+\infty, r_{n+1} / r_{n} \rightarrow 1$ as $n \rightarrow+\infty$. This is in fact the case. We have only to take $r_{n}=x_{n} \sec \delta$, where $x_{n}$ is the sequence mentioned in (56), and to observe that $\log |f(-x+i y)| \geqq \log |f(-x)|$ since

$$
\left|\left(1+\frac{-x+i y}{a_{n}}\right) e^{(x+i y) / a_{n}}\right| \geqq\left|\left(1-\frac{x}{a_{n}}\right) e^{x / a_{n}}\right|
$$

for all $a_{n}(>0)$.

In conclusion we should point out that this theorem could evidently be extended to the case $3 / 2<\rho<2$, if an alternative method of obtaining (59) can be found; for, if so, Lemma 2 can be used for the "gap-filling" argument.

\section{REFERENCES}

1. N. A. Bowen, Quart. J. Math. Oxford ser. vol. 19 (1948) pp. 90-100.

2. M. Heins, Ann. of Math. (1) vol. 49 (1948) pp. 200-213.

3. R. Nevanlinna, Eindeutige analytische Funktionen, Berlin, 1936. 1929.

4. - Le théorème de Picard-Borel et la thèorie des fonctions meromorphes, Paris,

5. R. E. A. C. Paley and N. Wiener, Fourier transforms in the complex domain, Amer. Math. Soc. Colloquium Publications, vol. 19, New York, 1934.

6. E. C. Titchmarsh, Proc. London Math. Soc. (2) vol. 26 (1926) pp. 185-200.

7. G. Valiron, Annales de Toulouse (3) vol. 5 (1914) pp. 117-257.

8. —, Thèses, Toulouse, 1914.

King's COLlege,

Aberdeen, Scotland. 